\title{
Transverse colon cancer occurring at a colostomy site 35 years after colostomy: a case report
}

\author{
Chiyo Maeda*, Eiji Hidaka, Mari Shimada, Shoji Shimada, Kenta Nakahara, Daisuke Takayanagi, Yusuke Takehara,
} Shumpei Mukai, Naruhiko Sawada, Fumio Ishida and Shin-ei Kudo

\begin{abstract}
Background: Carcinomas occurring at colostomy sites are rare, and most of these are metachronous colorectal cancers. The median time between colostomy and development of a carcinoma at a colostomy site is 22 years, which exceeds the length of the recommended follow-up period. We report a rare case of a carcinoma of the transverse colon occurring at a colostomy site in a patient without a history of colorectal cancer.

Case report: An 89-year-old woman presented with a tumor occurring at a colostomy site. Thirty-five years previously, she had undergone a transverse loop colostomy for an iatrogenic colon perforation that occurred during left ureteral lithotomy. Upon physical examination, the patient had a hard nodule measuring $3 \mathrm{~cm}$ at the colostomy site. A biopsy of the nodule suggested adenocarcinoma, and the preoperative diagnosis was transverse colon cancer. A laparotomy was performed via a peristomal incision with 5-mm skin margins, and the tumor was covered by a surgical glove to avoid any tumor seeding. The colon was separated from the tumor by $5-\mathrm{cm}$ margins, and the specimen was removed en bloc. An end colostomy was constructed to a new site on the right side of the abdomen. The deficit in the abdominal wall was repaired, and the skin was closed via a purse-string suture. The final diagnosis of the stoma tumor was transverse colon cancer (T2, NO, MO, stage I). One year and five months after surgery, there was no evidence of recurrence.
\end{abstract}

Conclusions: The occurrence of carcinomas at colostomy sites in patients without a history of colorectal cancer is rare. It is important to train ostomates to monitor the stoma for possible tumor recurrence.

Keywords: Colostomy site, Ostomate, Colorectal cancer

\section{Background}

Carcinomas occurring at colostomy sites are rare, with only 12 cases previously reported in the English literature (Table 1); most of these are metachronous colorectal cancers. Cases without malignant potential, such as those involving colorectal cancer or ulcerative colitis, are very rare. Herein, we describe a case of an 89-year-old woman who presented with a colostomy tumor 35 years after undergoing a transverse colostomy for iatrogenic colon perforation, and we review the literature related to this malignancy.

\footnotetext{
* Correspondence: maechiyo@hotmail.co.jp

Digestive Disease Center, Showa University Northern Yokohama Hospital, 35-1, Chigasaki-Chuo, Tsuzuki-ku, Yokohama 224-8503, Japan
}

\section{Case presentation}

An 89-year-old Japanese woman with a 1-month history of stoma bleeding was referred to our hospital. She had undergone a transverse loop colostomy 35 years previously for an iatrogenic colon perforation that occurred during left ureteral lithotomy. Upon physical examination, the patient was found to have a hard nodule at the colostomy site, measuring $3 \mathrm{~cm}$ (Figure 1). A biopsy of the nodule suggested adenocarcinoma. Laboratory data revealed anemia and renal dysfunction. Serum carcinoembryonic antigen and carbohydrate antigen 19-9 levels were not elevated. Staging computed tomography showed a 3-cm tumor adjacent to the stoma with no invasion into the abdominal muscle. There was no evidence of metastatic disease. Colonoscopy via the stoma revealed no other lesions in the colon or rectum. On the basis of our findings, the preoperative diagnosis of the stoma tumor was transverse colon cancer (T2, N0, M0, 
Table 1 Twelve previously reported cases of carcinomas at a stoma site

\begin{tabular}{|c|c|c|c|c|c|c|c|c|c|}
\hline Author & Year & Age/sex & Past history & Term (years) & $\begin{array}{l}\text { Chief } \\
\text { complaint }\end{array}$ & Size $(\mathrm{cm})$ & Depth & Histology & Prognosis \\
\hline Morgan & 1966 & $76 / F$ & Ulcerative colitis & $\begin{array}{l}31 \text { years after } \\
\text { colostomy }\end{array}$ & Bleeding & $7.4 \times 6.4$ & SI (skin) & Adenocarcinoma & - \\
\hline Didolkar & 1975 & $42 / F$ & Rectal carcinoma & $\begin{array}{l}32 \text { years after } \\
\text { colostomy }\end{array}$ & Stoma ulcer & 3 to 4 & - & Basal cell carcinoma & Alive (2.5 years) \\
\hline Takami & 1983 & $53 / \mathrm{M}$ & Rectal carcinoma & 19 years after APR & Tumor & 16 & SI (skin) & Mod, muc & Alive (2 years) \\
\hline Kusunoki & 1996 & $63 / M$ & Ulcerative colitis & $\begin{array}{l}6 \text { years after } \\
\text { colostomy }\end{array}$ & - & - & MP & Mod, muc & Alive (5 years) \\
\hline Shibuya & 2002 & $57 / M$ & Rectal carcinoma & 8 years after APR & Stoma stenosis & $6 \times 4$ & SI (skin) & Mod & Alive (4 years) \\
\hline Townley & 2005 & $57 / F$ & Rectal carcinoma & 5 years after APR & Tumor & 3 & Dukes A & Adenocarcinoma & Alive (6 months) \\
\hline Papaziogas & 2006 & $77 / F$ & Rectal carcinoma & $\begin{array}{l}3 \text { months after } \\
\text { Hartmann }\end{array}$ & Tumor & 2.5 & - & Adenocarcinoma & Death (1 year) \\
\hline Chintamani & 2007 & $30 / M$ & Rectal carcinoma & 6 years after APR & Stoma stenosis & - & T4N1 & Por, sig & Death (4 years) \\
\hline Vijayasekar & 2008 & $61 / F$ & Rectal carcinoma & 14 years after APR & Tumor & - & SI (subcutaneous) & Well & - \\
\hline Okamoto & 2009 & $67 / M$ & Rectal carcinoma & 15 years after APR & Tumor & $8 \times 10.5$ & SI (muscle) & Well & - \\
\hline Sabater-Marco & 2013 & $61 / M$ & $\begin{array}{l}\text { Lung/rectal } \\
\text { carcinoma }\end{array}$ & 6 years after APR & Tumor & - & - & Large cell carcinoma & Death (3 months) \\
\hline Maurra & 2014 & $75 / F$ & Sigmoid volvulus & $\begin{array}{l}50 \text { years after } \\
\text { Hartmann }\end{array}$ & Tumor & - & SI (muscle) & Well, muc & Alive (8 years) \\
\hline Our case & 2014 & $89 / F$ & $\begin{array}{l}\text { latrogenic } \\
\text { perforation }\end{array}$ & $\begin{array}{l}35 \text { years after } \\
\text { colostomy }\end{array}$ & Bleeding & 3.3 & MP & Mod & Alive (11 months) \\
\hline
\end{tabular}

F: female, M: male, APR: abdominoperineal resection, mod: moderately differentiated adenocarcinoma, muc: mucinous adenocarcinoma, Sl: infiltration to other organ, MP: proper muscle. 


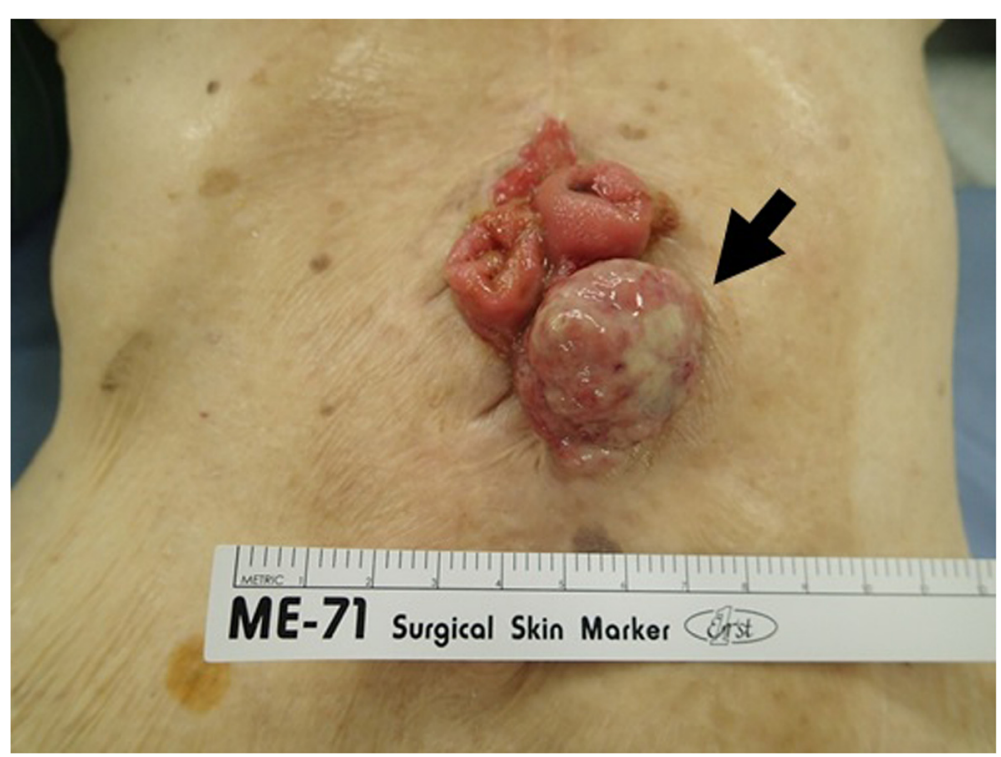

Figure 1 Macroscopic findings on admission. The patient had a hard nodule that measured $3 \mathrm{~cm}$ at the colostomy site. The arrows indicate the tumor.

stage I). We concluded that colectomy and repositioning of the colostomy site were appropriate for this patient. Although we thought stoma closure was possible, the patient and her family were opposed to this strategy because they were concerned about anal function.

Under general anesthesia, a laparotomy was performed via a peristomal incision with 5-mm skin margins, and the tumor was covered using a surgical glove to avoid tumor seeding. Adhesion around the stoma was not strong, and there was no evidence of peritoneal dissemination. Only pericolic lymph nodes were dissected. The colon was separated from the tumor by 5-cm margins, and the specimen was removed en bloc (Figure 2). An end colostomy was constructed to a new site on the right side of the abdomen. The deficit in the abdominal wall was repaired, and the skin was closed via a purse-string suture. The operative time was 102 $\mathrm{min}$, and blood loss was $52 \mathrm{~mL}$. Macroscopic examination showed complete excision of the tumor with clear margins (Figure 3).

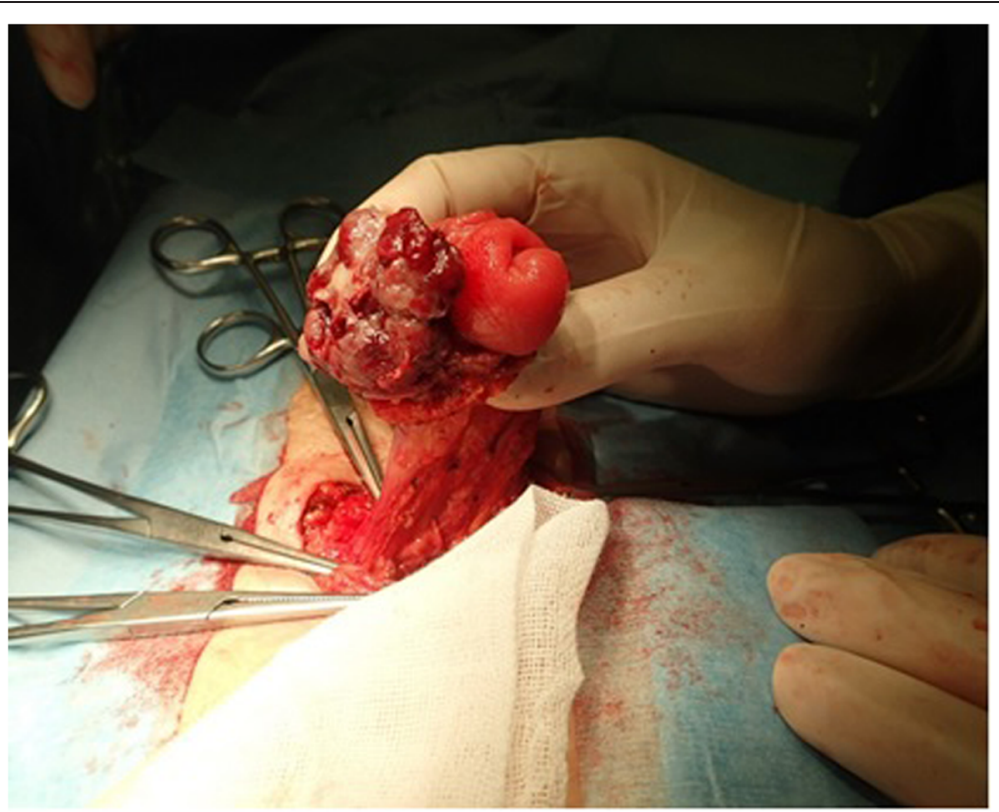

Figure 2 Surgical findings. The tumor was removed en bloc. 


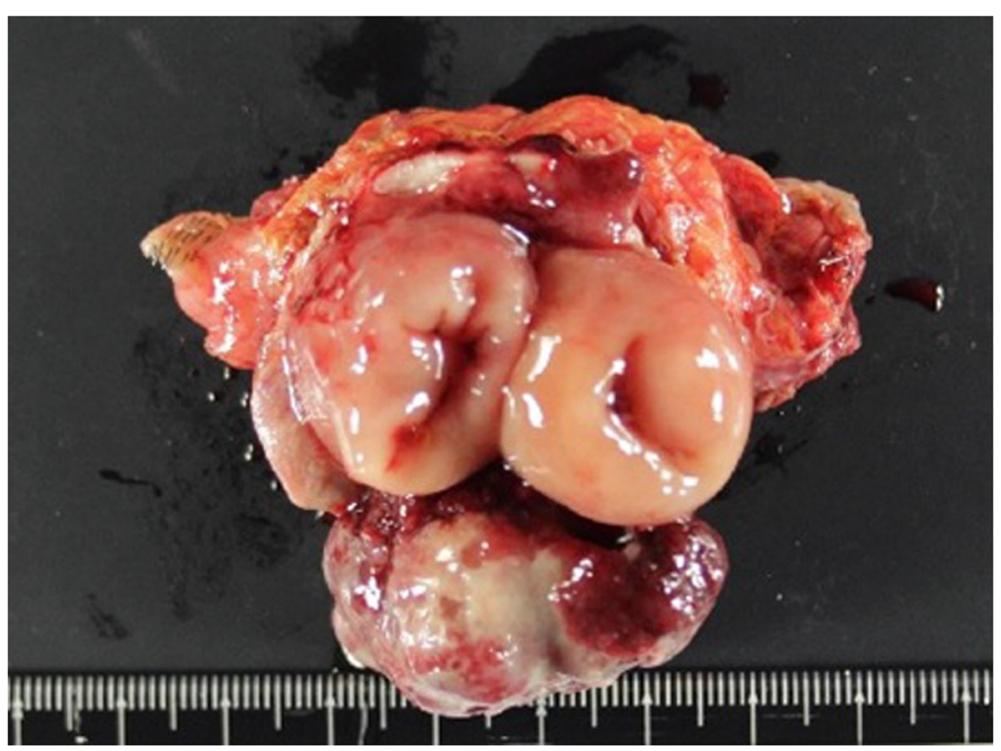

Figure 3 Macroscopic findings. Macroscopic examination showed complete excision of the tumor with clear margins.

The patient was discharged 8 days after surgery. Her postoperative course was uneventful, and she recovered without any complications. One year and five months after surgery, there is no evidence of recurrence.

Microscopic examination of the tumor showed moderately differentiated adenocarcinoma mixed with welldifferentiated adenocarcinoma (Figure 4). The tumor extended into the proper muscle layer and directly invaded the skin. There was no lymph node metastasis.
The final diagnosis of the stoma tumor was transverse colon cancer (T2, N0, M0, stage I).

The study protocol was approved by the ethics committee of Showa University Northern Yokohama Hospital. The study was conducted according to the Declaration of Helsinki.

\section{Discussion}

Primary adenocarcinoma arising at a colostomy site is rare. The first case was reported by Morgan in 1966 [1]

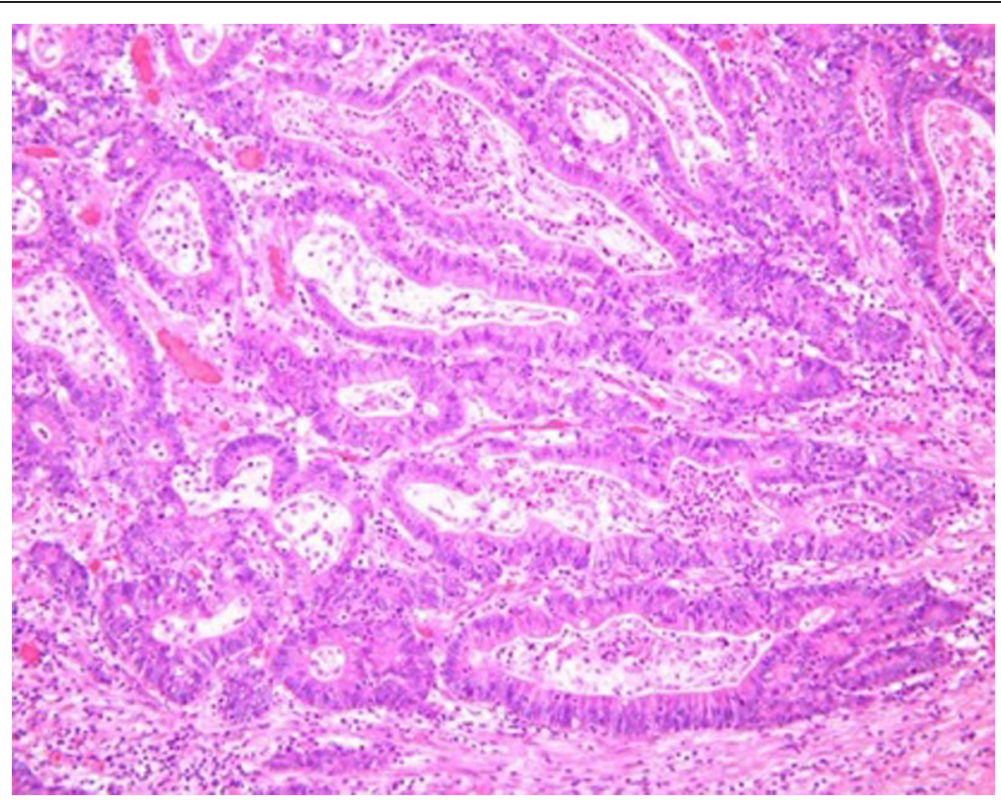

Figure 4 Microscopic findings. Microscopic examination showed moderately differentiated adenocarcinoma mixed with well-differentiated adenocarcinoma. 
and involved a patient whose underlying disease was ulcerative colitis. The 12 cases reported previously involved patients with metachronous colon cancer (eight patients), ulcerative colitis (two patients), metachronous lung cancer (one patient) [2], and a sigmoid volvulus (one patient) [3] (Table 1). Malignant potential was lacking only in the sigmoid volvulus case and in the case reported here. Cancers arising near stomas typically show skin metastasis [4] and include metachronous cancers. Immunopathological staining of cytokeratin facilitates the differential diagnosis [2].

Cutaneous metastasis of colon cancers to operation scars after cancer resection accounts for $0.6 \%$ of cases of recurrence [5]. The mean interval to the development of skin metastasis after colon cancer diagnosis is 4.9 years [6]. The average survival of patients with skin metastasis is 7.5 months after diagnosis [7], whereas the median time to the development of a carcinoma at a colostomy site after the initial operation is 22 years [8]. Most stoma site carcinomas are diagnosed as advanced cancers. The Japanese national guidelines [9] recommend a 5-year follow-up period after colorectal cancer resection. Thereafter, self-observation of the stoma by the patient is very important for the early detection of cancer. In our hospital, nurses who specialize in stoma care train ostomates in the methods of self-care and selfobservation at the outpatient clinic for several years. The patient in the case reported here had not received this training and did not know about the possibility of a carcinoma arising at the stoma site. It was also difficult for her to recognize the tumor because it grew slowly over several years. Thus, it is important to train ostomates in regard to self-observation of the stoma.

Of the 12 previously reported cases of adenocarcinomas arising at colostomies, six involved patients with T4 colon cancer that invaded the skin or subcutaneous tissue (pkpk). In the literature, it is not clear whether tumors directly invade the skin or penetrate the colon serosa. In the patient described in this report, tumor invasion included the proper muscle layer, with direct invasion of the skin. The depth of colostomy site cancer needs to be clarified in terms of direct invasion or invasion through the colon serosa.

Our patient had no history of cancer, radiation therapy, or ulcerative colitis; the case reported here therefore represents an extremely rare case of colostomy site carcinoma that did not have malignant potential. The etiology in this case is unclear. The carcinoma may have resulted from physical stimulation caused by clothing or unexpected compression, or by chemical stimulation, such as that resulting from enterobacteria or bile acids in stools [10]. On the other hand, stoma site cancers could also occur coincidentally. The incidence of metachronous colorectal cancer after abdominoperineal resection for rectal cancer is $2.2 \%$ [11]. Most metachronous tumors (51\%) are located in the left hemicolon, as are most stomas after colorectal cancer. Therefore, in this case, it seems that a colon cancer occurred coincidentally at the stoma site; however, physical stimulation or chemical stimulation could promote their occurrence.

In surgical operations, it is best not to expose the tumor. Cutaneous recurrence at the stoma closure suture after 5 years has been reported [12]. In this case, the tumor was not exposed because it was covered by a surgical glove. Because surgical site infection is very common after stoma closure, a purse-string skin closure [13], which reduces wound infection [14], was performed in this case.

The number of reports describing ileostomy site carcinoma is greater than the number of reports describing colostomy site carcinoma [15]. En bloc resection of the ileostomy, wide resection of the adjacent anterior abdominal wall, and transposition of the stoma to a new site have been shown to provide the best prognosis for a patient diagnosed with adenocarcinoma following ileostomy [16].

The mechanism of cancers arising at ileostomy sites is also speculative. Most grow at the mucocutaneous junction [17]. Previous reports have proposed that physical trauma or chemical or physical irritation predisposes the ileal mucosa to colonic metaplasia, dysplasia, and malignant change. Although there are many more colorectal cancer patients than small intestine cancer patients, there are fewer case reports of colostomy site carcinomas than ileostomy site carcinomas. The mechanism of cancer development at ileostomy sites differs from that at colostomy sites. Chronic metaplasia and dysplasia were found in the ileal mucosa adjacent to tumors in patients with ulcerative colitis and familial adenomatous polyposis [15], suggesting that the malignant potential might be high in patients with these underlying diseases.

\section{Conclusions}

Primary adenocarcinomas arising at colostomy sites are rare. Colostomy site carcinomas may occur after the postoperative follow-up period has ended. In order to facilitate early detection, it is important to train ostomates to monitor the stoma for possible tumors.

\section{Consent}

Written informed consent for the publication of this case report and any accompanying images was obtained from the patient's family. A copy of the written consent is available for review by the Editor-in-Chief of this journal. 


\section{Competing interests}

The authors declare that they have no competing interests.

\section{Authors' contributions}

CM and EH wrote the manuscript. SS and KN collected the data. SM, DT, YT, SM, NS, Fl, and SK performed literature searches. All authors read the manuscript and provided suggestions. All authors have approved the final version of the manuscript and agree to be accountable for all aspects of the report.

Received: 4 January 2015 Accepted: 24 April 2015

Published online: 06 May 2015

\section{References}

1. Morgan MN. Carcinoma in a caecostomy in longstanding ulcerative colitis. Proc R Soc Med. 1966;59:427.

2. Sabater-Marco V, Garcia-Garcia JA, Roig-Vila JV. Basaloid large cell lung carcinoma presenting as cutaneous metastasis at the colostomy site after abdominoperineal resection for rectal carcinoma. J Cutan Pathol. 2013;40:758-64.

3. Mourra N, Bataillon G, Lesurtel M. Fungating mass occurring at a colostomy site 50 years after colectomy for inflammatory condition. Gastroenterology. 2014;146:e1-2.

4. Vijayasekar C, Noormohamed V, Cheetham MJ. Late recurrence of large peri-stomal metastasis following abdomino-perineal resection of rectal cancer. World J Surg Oncol. 2008;6:96-100.

5. Reilly WT, Nelson H, Schroeder G, Wieand HS, Bolton J, O'Connell MJ. Wound recurrence following conventional treatment of colorectal cancer. A rare but perhaps underestimated problem. Dis Colon Rectum. 1996;39:200-7.

6. Alexandrescu DT, Vaillant J, Yahr LJ, Kelemen P, Wiernik PH. Unusually large colon cancer cutaneous and subcutaneous metastases occurring in resection scars. Dermatol Online J. 2005;11:22.

7. Saeed S, Keehn CA, Morgan MB. Cutaneous metastasis: a clinical, pathological, and immunohistochemical appraisal. J Cutan Pathol. 2004;31:419-30.

8. Okamoto N. Metachronous multiple carcinomas arising at a colostomy site 18 years after abdominoperineal resection. Int J Colorectal Dis. 2009;24:1481.

9. Watanabe T, Itabashi M, Shimada Y, Tanaka S, Ito Y, Ajioka Y, et al. Japanese Society for Cancer of the Colon and Rectum (JSCCR) guidelines 2010 for the treatment of colorectal cancer. Int J Clin Oncol. 2012;17:1-29.

10. Shibuya T, Uchiyama K, Kokuma M, Shioya T, Watanabe $Y$, Moriyama Y, et al. Metachronous adenocarcinoma occurring at a colostomy site after abdominoperineal resection for rectal carcinoma. J Gastroenterol. 2002;37:387-90.

11. Zilli L, Pietroiusti M, Bertario L. Colonoscopy in ostomy patients. Results at the first postoperative examination. Dis Colon Rectum. 1987;30:687-91.

12. Chintamani Singhal V, Bansal A, Bhatnagar D, Saxena S. Isolated colostomy site recurrence in rectal cancer-two cases with review of literature. World J Surg Oncol. 2007;5:52-6.

13. Banerjee A. Pursestring skin closure after stoma reversal. Dis Colon Rectum. 1997:40:993-4

14. Camacho-Mauries D, Rodriguez-Díaz JL, Salgado-Nesme N, González QH, Vergara-Fernández $\mathrm{O}$. Randomized clinical trial of intestinal ostomy takedown comparing pursestring wound closure vs conventional closure to eliminate the risk of wound infection. Dis Colon Rectum. 2013;56:205-11.

15. Quah HM, Samad A, Maw A. lleostomy carcinomas a review: the latent risk after colectomy for ulcerative colitis and familial adenomatous polyposis. Colorectal Dis. 2005;7:538-444.

16. Carey PD, Suvarna SK, Baloch KG, Guillou PJ, Monson JR. Primary adenocarcinoma in an ileostomy: a late complication of surgery for ulcerative colitis. Surgery. 1993;113:712-5.

17. lizuka T, Sawada T, Hayakawa K, Hashimoto M, Udagawa H, Watanabe G. Successful local excision of ileostomy adenocarcinoma after colectomy for familial adenomatous polyposis: report of a case. Surg Today. 2002;32:638-41.

\section{Submit your next manuscript to BioMed Central and take full advantage of:}

- Convenient online submission

- Thorough peer review

- No space constraints or color figure charges

- Immediate publication on acceptance

- Inclusion in PubMed, CAS, Scopus and Google Scholar

- Research which is freely available for redistribution

Submit your manuscript at www.biomedcentral.com/submit 